\title{
CALIDAD DE VIDA LABORAL Y ESTRATEGIAS DE MEJORA DEL TRABAJO DE LA ENFERMERÍA DOCENTE
}

\author{
QUALITY OF WORKING LIFE AND STRATEGIES FOR IMPROVING \\ THE WORK OF NURSE EDUCATORS
}

\section{QUALIDADE DE VIDA NO TRABALHO E ESTRATÉGIAS PARA MELHORAR O TRABALHO DO ENSINO DE ENFERMAGEM}

\author{
Denisse Parra-Giordano* \\ VANDA AndrÉs Felli** \\ María AngÉlica Saldías Fernández*** \\ Daniela Pinto-Galleguillos**** \\ Patricia Soto Malabrigo ${ }^{* * * * *}$
}

\begin{abstract}
RESUMEN
Objetivo: Identificar los factores laborales que influyen en la calidad de vida laboral de los enfermeros y enfermeras docentes y construir estrategias para mejorarla. Material y Método: Estudio descriptivo, exploratorio, cualitativo, desarrollado con enfoque de epidemiología crítica, realizado en una escuela de enfermería en Chile. Los sujetos de investigación corresponden a 17 enfermeras y enfermeros docentes, a los que se les realizaron entrevistas individuales semiestructuradas y 9 participaron en un grupo focal. Se contó con la aprobación del Comité de Ética de Investigación de la unidad académica. Resultados: Respecto a la categoría Calidad de vida se establecen las subcategorías: Relaciones humanas favorables, Ambiente favorable, Bienestar - Satisfacción y Estrés. En la categoría Estrategias de mejora, las subcategorías: Desde la empresa y Desde el trabajador. Conclusiones: Los enfermeros y enfermeras que se desempeñan en docencia ven afectada su calidad de vida por factores laborales, de tal forma que se deben establecer estrategias de mejora desde la empresa y el individuo.
\end{abstract}

Palabras clave: Calidad de Vida; Práctica del Docente de Enfermería; Condiciones de Trabajo; Salud Laboral; Empleo.

*Dra. en Ciencias de la Salud, Departamento de Enfermería, Facultad de Medicina, Universidad de Chile, Santiago, Chile. ORCID: 0000-0002-9439-2679. Email: denisseparrag@gmail.com Autor de correspondencia.

** Dra. en Enfermería, Escola de Enfermagem, Universidade de São Paulo, São Paulo, Brasil. ORCID: 0000-0001-7250-4353. Email: vandaeli@usp.br

${ }^{* * *} \mathrm{Mg}$. en Ciencias Políticas, Departamento de Enfermería, Facultad de Medicina, Universidad de Chile, Santiago, Chile. ORCID: 0000-0002-2278-9124. Email: mangelica.saldias@gmail.com

****Enfermera, Programa de Microbiología y Micología, Facultad de Medicina, Universidad de Chile, Santiago, Chile. ORCID: 0000-0003-4770-3419. Email: dpintogalleguillos@gmail.com

******Mg. en Gerencia y Políticas Públicas, Departamento de Enfermería, Facultad de Medicina, Universidad de Chile, Santiago, Chile. ORCID: 0000-0002-0130-5995. Email: patriciaysabelsoto@hotmail.com 


\section{ABSTRACT}

Objective: To identify the occupational factors affecting the quality of working life of nurse educators and design strategies to improve it. Material and Method: Descriptive, exploratory, qualitative study, developed with a focus on critical epidemiology, conducted at a nursing school in Chile. The research subjects corresponded to 17 nurse educators, who were given individual semi-structured interviews and 9 participated in a focus group. The research was approved by the Research Ethics Committee of the institution. Results: Regarding the category Quality of Life, the following subcategories were established: Positive Human Relations, Favorable Environment, Well-being/Satisfaction and Stress. In the category Improvement Strategies, the subcategories From the Employer and From the Worker emerged. Conclusions: Nurses who work as educators see their quality of life affected by occupational factors, so that improvement strategies are needed from the employing institution as well as from the worker.

Key words: Quality of Life; Nursing Faculty Practice; Working Conditions; Occupational Health; Employment.

\section{RESUMO}

Objetivo: Identificar os fatores trabalhistas que influenciam a qualidade de vida no trabalho do professor enfermeiro e construir estratégias para melhorá-lo. Material e Método: Estudo descritivo, exploratório, qualitativo, desenvolvido com foco em epidemiologia crítica, realizado em uma Escola de Enfermagem do Chile. Os sujeitos da pesquisa correspondem a 17 docentes de enfermagem, que foram submetidos a entrevistas individuais semiestruturadas e nove participaram de um grupo focal. Foi obtida a aprovação do Comitê de Ética em Pesquisa da unidade acadêmica. Resultados: Em relaçáo à categoria qualidade de vida, foram estabelecidas as subcategorias: relações humanas favoráveis, ambiente favorável, bem-estar - satisfação e estresse. Na categoria estratégias de melhoria, as subcategorias: Desde a empresa e Desde o trabalhador. Conclusóes: Os enfermeiros que atuam no ensino veem sua qualidade de vida afetada pelos fatores de trabalho, de maneira que a dialética nesse trabalho exige o estabelecimento de estratégias de melhoria por parte da empresa e do indivíduo.

Palavras-chave: Qualidade de Vida; Prática do Docente de Enfermagem; Condiçóes de Trabalho; Saúde do Trabalhador; Emprego.

Fecha de recepción: 24/01/2020

Fecha de aceptación: 16/08/2020

\section{INTRODUCCIÓN}

Comprender la enfermería como un trabajo implica aceptar cómo su ejercicio establece las prácticas de salud de los enfermeros y enfermeras, impactando en su Calidad de Vida Laboral (CVL) y en las prácticas de autocuidado respectivas ${ }^{(1)}$. El trabajo, además de ser una actividad con fines productivos que genera recursos útiles para costear la vida, determina la posición social y otorga sentido de pertenencia e identidad ${ }^{(2)}$. El ambiente de trabajo se ve afectado por la dinámica del desarrollo humano y la interacción entre factores físicos, sociales y gerenciales, ante lo cual es importante considerar la satisfacción y el bienestar, así como también la insatisfacción y aflicción que impactan en el proceso de salud - enfermedad y en la CVL ${ }^{(3)}$.

Mirado así, el concepto de CVL es cada vez más utilizado como una métrica del bienestar de los trabajadores, donde se interrelacionan diferentes $\operatorname{aspectos}^{(4)}$, que logran conformarse tomando en cuenta el trabajo que desempeńa cada persona, por medio de la reproducción social que determina su inserción social desde la perspectiva histórica ${ }^{(5)}$. Así nace el concepto de Calidad de Vida Laboral (CVL) o Calidad de Vida en el Trabajo, donde el ambiente y las condiciones de trabajo favorables protegen y promueven la satisfacción de los trabajadores ${ }^{(6)}$.

La CVL de los profesionales del área de la salud debería ser óptima al considerar los conocimientos y los medios necesarios para prevenir riesgos y realizar acciones de autocuidado en todas las 
esferas del ser humano(7), sin embargo, diversos estudios demuestran que dentro de este grupo de trabajadores, enfermería, entre otras problemáticas, ha desarrollado problemáticas asociadas a la falta de acciones de cuidado ${ }^{(7-10)}$, posiblemente asociadas a riesgos y condiciones laborales, que implican una carga mental que incide en la CVL y en la calidad de atención que brindan ${ }^{(11)}$.

En el contexto del trabajo de enfermería en ambientes académicos, los desafíos que subyacen a este quehacer surgen de las relaciones que se dan entre la educación superior, la sociedad, las demandas del mercado laboral y las transformaciones mundiales, y para resolverlos se requiere una articulación permanente entre la trayectoria profesional y la formación continuada de los académicos ${ }^{(4)}$, desarrolladas bajo el modelo hegemónico predominante, que impone las exigencias de la alta competencia, propias del mercado capitalista. Es por esto que la CVL de este profesional se ve afectada por el desempeño de su labor ${ }^{(2,12)}$, haciéndose imprescindible evaluar sus lugares de trabajo ${ }^{(11)}$. En este ámbito, es importante que el enfermero docente cuente con un elevado grado de satisfacción, pues ello repercute en la eficiencia del profesional sobre las tareas que desempeña ${ }^{(13)}$.

En la actualidad, por el aumento de la intención de estudiar la carrera de Enfermería, hay una falta de docentes de esa disciplina ${ }^{(11,14)}$, y las consecuencias que esto puede generar en la CVL de sus académicos ha sido escasamente estudiada ${ }^{(3)}$ 14, 15). En este contexto, algunos autores destacan que el potenciar la formación académica continua y el rediseño curricular según las demandas del mercado laboral, además de trabajar para mejorar aspectos del equilibrio vida-trabajo, son elementos a considerar si se quiere potenciar la calidad del trabajo realizado y elevar el grado de satisfacción con la actividad realizada. Con ello también se contribuye adecuadamente al desarrollo social, económico y cultural, porque se atiende a las necesidades de los trabajadores de enfermería, desde un punto de vista biopsicosocial ${ }^{(9,16-18)}$.

De cara al desarrollo del trabajo de enfermería docente en el mercado neoliberal actual, se reconoce la necesidad de una reflexión-acción en este tema, investigando desde el paradigma crítico para lograr una transformación social que mejore sus condiciones de trabajo ${ }^{(19)}$. Un promotor fundamental de la epidemiología crítica es Naomar Almeida Filho, quien sostiene que este abordaje puede desarrollarse tanto desde el diseño cualitativo como cuantitativo ${ }^{(20)}$.

Frente a estos antecedentes, se considera necesario desarrollar un estudio que aborde la calidad de vida de dicha realidad laboral, desde una perspectiva cualitativa, a partir de la subjetividad de los trabajadores, centrado en captar los factores laborales que influyen en la calidad de vida de los enfermeros docentes y construir estrategias para mejorarla.

\section{MATERIAL Y MÉTODO}

Tipo de estudio: Cualitativo, exploratorio y descriptivo, desarrollado con foco en la epidemiología crítica. El escenario fue la Escuela de Enfermería de la Universidad de Chile, fundada en 1906, acreditada por siete años (máximo otorgable), otorga el grado de Licenciado/a en Enfermería y el título de Enfermera/o y su último proceso de innovación curricular fue en el ańo $2013^{(21)}$.

Participantes: La población de estudio estuvo conformada por todos los docentes de enfermería, que totalizaban, al 2014, 52 personas. Se aplicaron los siguientes criterios de selección: jornada de trabajo 22, 33 o $44 \mathrm{~h}$ por semana, ejercicio como enfermera docente por seis o más meses. Se excluyeron a personas que estuvieran de vacaciones o licencia médica. De esta forma fueron 25 los sujetos susceptibles de participación.

Recolección de datos: Conforme a la revisión de la literatura se establecieron dos categorías de estudio: Calidad de vida laboral y Estrategias de mejora en el trabajo. En base a estas categorías se confeccionaron las 2 preguntas guía: ¿Qué factores laborales del enfermero docente influyen en su calidad de vida laboral? ¿Qué estrategias ustedes proponen para mejorar su calidad de vida en el trabajo?

Procedimiento: Se invitó a participar a los 25 docentes y todos aceptaron participar en las entrevistas. Conformea su respuestay disponibilidad de tiempo, se acordaron las entrevistas individuales, desarrolladas por la investigadora principal con relación laboral previa con los participantes. Se aplicaron las preguntas guía llegando a entrevistar a 17 sujetos hasta la saturación del objeto de estudio. 
Para las entrevistas, que tuvieron una duración de 15 a 32 min, se habilitó una sala en la Escuela de Enfermería señalada, con total privacidad, durante el mes de abril de 2014. Las entrevistas fueron grabadas y posteriormente transcritas integralmente para orientar el análisis.

Análisis y tratamiento de los datos: Los datos fueron sometidos a análisis de contenido según Bardin $^{(22)}$, teniendo en cuenta las categorías predefinidas. Conforme al análisis de la categoría Calidad de vida laboral se extraen cuatro subcategorías: Relaciones humanas favorables, Ambiente favorable, Bienestar-satisfacción y Estrés. De la categoría Estrategias de mejora en el trabajo se captaron dos subcategorías: Desde la empresa y Desde el trabajador. Para la presentación de datos se identificó a los entrevistados con la letra E, y se especificó el número de cada entrevista conforme al orden en que se desarrollaron.

Aspectos de rigor y éticos: Para asegurar la validez de la investigación, esta fue desarrollada según los criterios de rigor de Guba y Lincoln ${ }^{(23)}$. Asimismo, éticamente el estudio fue aprobado por el Comité de Ética de Investigación en Seres Humanos de la Facultad de Medicina de la Universidad de Chile ( $\left.\mathrm{N}^{\circ} 166-2013\right)$, autorizado por la dirección de la escuela y todos los sujetos firmaron el Consentimiento Informado.

\section{RESULTADOS}

\section{Categoría Calidad de Vida Laboral (CVL)}

Subcategoría Relaciones humanas favorables. La forma de relacionarse en el trabajo impacta en la CVL, citadas de forma genérica y la necesidad de su existencia en el trabajo en equipo:

"La calidad de vida va relacionado con hartos aspectos, sobre todo con... creo que a nivel laboral va muy relacionado con lo que es las relaciones humanas, ja jajá (se rie), como te mencionaba antes, mi calidad de vida hasta el momento no ha sido negativa aca" (E8). "Calidad de vida para mi en el trabajo es poder trabajar en equipo, porque uno no puede trabajar solo, en docencia es imposible" (E6).

Subcategoría Ambiente favorable. Los docentes apuntan al ambiente como un aspecto que favorece la calidad de vida en el trabajo, tomando el equilibrio entre la exigencia y la demanda de tareas:

"El significado de la calidad de vida en mi trabajo...acá, mira yo creo que somos como hartas profes como bien inquietas creo yo, entonces en el trabajo creo tener el ambiente laboral propicio" (E4).

"Pero en el fondo yo vengo de un trabajo más exigente que este en términos de horario, en términos de estrés, en términos de cómo, politicamente hablando y pa' mi fue un buen cambio" (E16).

Subcategoría Bienestar-satisfacción. Durante las entrevistas los enfermeros docentes reconocen el gusto por el trabajo realizado, a tal punto de ocupar tiempo personal para desarrollar sus funciones de modo consciente, esto es, que ellos son obsesivos en el trabajo, lo cual, conforme al análisis, es una característica de la profesión:

"Mira el tema calidad de vida es un tema complejo, porque en realidad las enfermeras como que no sabemos lo que es la calidad de vida", "la calidad de vida yo la relaciono con el bienestar obviamente, con la felicidad que tú tengas en tu vida, y claro yo estoy muy contenta, estoy feliz de estar acá, pero resulta que yo me reconozco una persona trabajólica, entonces como yo me reconozco una persona trabajólica a mi me acomoda este sistema de vida"(E9).

"Yo tengo estudiantes que son egresados y que me mandan mails y me dicen "profe yo nunca me voy a olvidar cuando usted me enseñó a puncionar", yo le digo no soy tu profe, somos colegas, entonces, eso es súper gratificante, yo creo que eso no tiene un valor económico, pero sin embargo cuando tú hablas de calidad de vida, eso para mi es súper valorable y no tiene precio"(E6).

Subcategoría Estrés. La calidad de vida en el trabajo es percibida como buena, incluso con la presencia de estrés y un propicio ambiente laboral. $\mathrm{Al}$ mismo tiempo, es muy importante que la docencia sea vista como un área de desarrollo de la profesión, con mayor calidad de vida. El estrés presenta una naturaleza doble al ser referido como un problema $y$, a la vez, promover otros problemas de salud:

"O sea que sea exigente, pero que a la vez también no te ahogue, que tengas los recursos para trabajar, tu oficina, tu espacio, las relaciones interpersonales con tus colegas, con tu jefatura, el tema del manejo del estrés" (E4). 


\section{Categoría Estrategias de mejora}

Subcategoría Desde la empresa. En esta subcategoría, el primer tema es la política institucional y el sistema contractual y colectivo. Los docentes refieren que es la empresa quien debe partir con la implementación de estrategias, estableciéndose la necesidad como prioritaria desde los lineamientos institucionales. Asimismo, debe ser colectivo, con la responsabilidad como grupo de trabajo de mejorar el ambiente laboral, promoviendo un trabajo en equipo, respetando los límites de los otros y las cargas de trabajo de cada uno, así como también, enseñando a los estudiantes para mejorar la CVL:

"Hayjornadas de autocuidado, nosotros deberíamos irnos una vez al año en una jornada a todo cohete, digamos, somos la mejor universidad, con ribetes internacionales y todo, $y$ sin embargo yo siento, $y$ de verdad que creo que hay poco cuidado hacia nosotras siendo que somos el capital, el capital que sostiene, el capital intelectual" (E15).

"El sistema contractual, porque yo encuentro que es injusto, que haya colegas que estén contratadas por honorarios, y otras colegas que estén, o sea, es un mix de cosas, entonces yo encuentro que es injusto que a algunos le estén pagando todas las imposiciones y todas las cosas, y a otras personas que no, uno dice, oye tienes acceso a ir a tal cosa, no, es que tú eres honorario no puedes hacer esto otro" (E1).

"También hay que tener una cierta unión colectiva, para lo que es ambiente laboral, no saco nada yo con querer que haya un comedor, si hay dos personas que tienen diferencias, no van a ir al comedor, al final lo transmite" (E8).

"Hay un colectivo que tiene que funcionar y tiene que funcionar como un equipo, yo también soy como de la camada nueva, aún más nueva que E3, y siento que en muchas de las asignaturas que trabajamos, lo hacemos como grupo, pero no somos un equipo y ahi también viene el protagonismo del liderazgo" (E14).

Se desprende como segundo tema la Infraestructura, por la necesidad de tener una habilitación de espacios adecuada para desarrollar el trabajo, es decir, lugares para almorzar, recibir a los estudiantes y realizar el trabajo completo durante la jornada, sin llevar asuntos pendientes al regreso a casa, que ocupan los tiempos de vida personal:

"Un comedor en verdad me parece una intervención que en verdad es muy necesaria, una necesidad sentida por todos" (E14).
El tercer tema dice relación con el Clima y las relaciones laborales, las cuales son reconocidas como elementos importantes para mejorar la CVL, contribuyendo con el trabajo en equipo, según las competencias de cada persona. De esta forma, se podrían fomentar los espacios para compartir entre los trabajadores y promover la mejora de las relaciones al interior del trabajo:

"Yo creo que es fundamental tener espacios comunes para poder interactuar", "es fundamental, o sea, las estrategias pueden venir de uno, pero si falta acá, o sea, fundamental, después vienen las prácticas, hay que llegar apurado a comer, para nuevamente estar a las dos de la tarde" (E2).

"Yo creo que eso podría ser una estrategia también, que desde los equipos se elija a quienes son los lideres de cada equipo" (E3).

"Felicidad organizacional, que eso tiene que ver con que nos juntemos no en el horario de trabajo, vivan los happy hours, vivan las reuniones que son para conocernos en el ámbito personal, porque en el fondo uno acá no sólo viene a aportar con su trabajo, uno pone parte de su persona y eso también se proyecta en sus estudiantes" (E14).

Subcategoría Desde el trabajador. Respecto a las estrategias de mejora desde el trabajador, el tema principal se refiere a estrategias personales. Los trabajadores mencionan que cada persona tiene la responsabilidad en mejorar su CV, es decir, cada persona es responsable de poner límites, manteniendo el equilibrio con el trabajo, conforme con la realidad individual de cada uno:

"Desde ya trabajar en forma preventiva, para tener un envejecimiento de calidad, se puede decir, cuando llegue a mayor de 60 años, o 65 años, entonces, enfocarlo hacia allá, ser una persona activa, ser una persona medianamente saludable, porque no vas a lograrlo cien por ciento, pero si tener un envejecimiento activo"...... "entonces en la medida de que yo puedo hacer intervenciones tanto con mis hijos como con mi familia para poder realmente llegar a tener una calidad de vida mejor a futuro" (E7).

\section{DISCUSIÓN}

Como se dijo, la calidad de vida laboral ha sido poco estudiada en docentes en Enfermería. En el presente estudio los docentes participantes 
identificaron que las relaciones humanas favorables, un ambiente positivo y el gusto por el trabajo realizado, influyen favorablemente en la CVL, así como también se reconoció la exposición a estrés como un problema en sí mismo y como un factor promotor de otros problemas de salud. Seguidamente se enunciaron estrategias para mejorar la CVL a nivel de empresa, donde se señaló a las instituciones como responsables de comenzar con la implementación de estrategias de manera prioritaria desde lineamientos institucionales y a nivel colectivo, resaltando la responsabilidad del grupo de trabajo para mejorar la CVL, así como también proveyendo de infraestructura adecuada; por otro lado, a nivel individual, se recalcó la responsabilidad personal como estrategia en la mejoría de la CVL.

En adición a lo anterior, llama la atención, en los discursos de los docentes, la escasez de relatos sobre problemas de salud. Este hecho puede estar relacionado a la falta de conocimiento y concientización sobre la relación trabajo-salud. Con todo, es posible inferir la insatisfacción y desmotivación de los docentes con las condiciones de trabajo. La satisfacción laboral es sentida por los docentes $^{(8,14,18)}$.

En lo que respecta a las relaciones humanas, los docentes de un estudio realizado en Fortaleza Brasil mencionan que la CVL tiene relación con todo tipo de vínculo social, económico o político y está relacionado con un buen equilibrio en la vida, tener buenas condiciones de vida y lograr satisfacción de las necesidades $^{(24)}$. También se señala que los conflictos con otras personas del trabajo impactan en la CVL, reconociendo que el proceso de trabajo docente no requiere del trabajo en equipo ${ }^{(25)}$. Contrario a esto último, en este estudio las relaciones humanas $y$ el trabajo en equipo se reconocen como un factor que contribuye en forma positiva a la CVL y concuerda con estudios que señalan que la falta de integración y el individualismo de las personas influye negativamente en la CVL ${ }^{(26)}$.

Es importante comprender que el trabajo extrapola las salas de clases o el acompañamiento en el campo clínico, debido a que el producto de este proceso de trabajo no es inmediato, pues la formación de estudiantes toma años. De esta forma, las gratificaciones no monetarias del trabajo también pueden ser recibidas años después, con los graduados.
En relación a los estudiantes, estos son reconocidos como un factor motivador en el trabajo ${ }^{(26)}$ y favorecen la CVL a través del proceso de enseñanza aprendizaje $\mathrm{e}^{(8)}$. En cuanto al ambiente, es interesante observar cómo la exigencia no es vista como algo negativo, más bien favorable a la CVL, tal vez porque la exigencia permite mostrar la capacidad de realización, mientras no sea demasiada. De la misma forma, diferentes estudios reconocen al ambiente de trabajo como una condición favorable de la CVL, aunque también se puede tornar desgastante por situaciones en el trabajo ${ }^{(8,24)}$. Con todo, se considera que el ambiente favorable es un incentivo para permanecer en la docencia ${ }^{(11)}$. Es primordial que los empleadores promuevan y mantengan un ambiente de trabajo saludable ${ }^{(11)}$, especialmente uno que esté basado en el cuidado de los demás. Así se puede retener a los docentes enfermeros y enfrentar la actual escasez de estos profesionales en la docencia.

En lo referente al bienestar-satisfacción, en varios estudios los docentes reconocen la extensión de la jornada y la sobrecarga en el proceso de trabajo del enfermero docente ${ }^{(8,26,27)}$, pero aun así refieren tener otras gratificaciones $y$, finalmente, logran obtener bienestar en el trabajo. Relacionado con esto, en un estudio realizado con trabajadores del sector público, en Colombia, la mayoría se reportó satisfecho o medianamente satisfecho en la dimensión bienestar ${ }^{(28)}$, entendiéndose entonces, que la satisfacción laboral es uno de los indicadores principales en la CVL de los profesionales de la $\operatorname{salud}^{(7)}$.

Se destaca que debido a que la mayoría de los trabajadores en esta área son de género femenino, condiciona a que tengan una carga mayor de trabajo por las actividades del hogar ${ }^{(4,24,26)}$, viviendo así entre el mundo del placer y del sufrimiento ${ }^{(8)}$. Un estudio en Nueva Zelanda refiere que aunque los enfermeros desean tener tiempo para sus familias o para realizar actividades de ocio, la carga de trabajo no se los permite por quedar físicamente agotados ${ }^{(9)}$. En el área asistencial, el profesional de enfermería se ve enfrentado a trabajos muy exigentes, por los sistemas de turnos o la alta demanda de cuidados de las personas enfermas y aunque el trabajo docente demanda tiempos extra o quita tiempos de la familia, comparativamente, el trabajo de entregar cuidados es todavía más estricto.

En Chile, el estrés es uno de los problemas más 
evidenciados en enfermeras ${ }^{(3)}$. Frente a esto, la extensión de la jornada, el llevar trabajo al hogar, las remuneraciones insuficientes ${ }^{(14,29)}$, la demanda de los estudiantes ${ }^{(8)}$ y la sobrecarga docente ${ }^{(24,29)}$, son reconocidas como factores que originan estrés y desgastan la CVL, así también la infraestructura y los materiales didáctico-pedagógicos insuficientes ${ }^{(8)}$.

Es importante mencionar que los docentes refieren que, producto del estrés sufrido en el trabajo, no consiguen cubrir sus necesidades de salud, lo que origina cansancio mental y fatiga generalizada ${ }^{(8)}$. Es importante señalar que aunque, en algún grado, este trabajo estresante tendría consecuencias negativas para la salud ${ }^{(25)}$, Breilh ${ }^{(2)}$ refiere que el estrés es un proceso mediador epidemiológico entre la vida y el trabajo y que no se reduce solo a la carga o subcarga mental en el centro laboral, porque es un proceso más complejo que involucra varias esferas de la vida del trabajador. Frente a esto, algunas nuevas generaciones de enfermeras, con 25 años en promedio, enfatizan lo importante que es tener un estilo de vida equilibrado entre lo personal y laboral ${ }^{(9)}$.

En relación a las Estrategias de mejora, en la literatura ya se han identificado las áreas críticas para un ambiente de trabajo saludable: salario, beneficios, carga de trabajo, ambiente universitario, preparación de roles y desarrollo profesional, becas, mercadeo y reconocimiento, apoyo institucional y liderazgo ${ }^{(11)}$; quedando en evidencia la necesidad de implementar intervenciones, por parte de la institución, en esas condiciones de trabajo del enfermero docente ${ }^{(10)}$.

En cuanto a la viabilidad de implementar estrategias relacionadas con la Política institucional y sistema contractual, podemos señalar que el Departamento de Enfermería estudiado, durante el año 2016 comenzó un proceso de Departamentalización que buscó introducir cambios, tanto en la planta física como en la organización de los recursos humanos, en el marco de plan de desarrollo estratégico de la Facultad de Medicina ${ }^{(21)}$ y del proceso de desarrollo institucional( ${ }^{(30)}$ de la universidad. Así, el año 2018, se eliminaron los contratos a honorarios, quedando todas estas personas con contrato a plazo fijo, conforme a la tarea específica que debían realizar. Este nuevo sistema contractual es un área mejorada, teniendo a todos los docentes con los mismos beneficios, tales como asistir a capacitaciones, inscribirse en el servicio de bienestar y acceder al bono de alimentación y transporte ${ }^{(31)}$, entre otros. Por otro lado, a pesar de encontrarse aprobado, desde hace años, un proyecto de ampliación del Departamento, que considera oficinas para la atención de estudiantes, salas de reuniones grupales y un comedor, este ha sido pospuesto debido a otras prioridades de gasto por parte de la Facultad, aunque la infraestructura es reconocida como táctica para mejorar la CVL por los mismos docentes.

Al respecto, hay estudios que señalan que hay instituciones que no generan instancias para promover el bienestar en el trabajo ${ }^{(8)}$, u otras en donde falta apoyo por parte de la administración ${ }^{(29)}$, aun cuando se sabe que si la institución no cuida a sus docentes, ofreciendo facilidades y escuchando sus necesidades, puede llevar a que los profesores dejen la institución en busca de una donde el conocimiento y la habilidad sean valorados ${ }^{(11)}$. Contrario a lo anterior, las instituciones deben fomentar la práctica de ejercicios físicos, mantener programas de divulgación de una alimentación saludable y ofrecer servicios educativos ${ }^{(26)}$.

En la gestión universitaria se debe considerar la generación de políticas que propicien espacios para desarrollar acciones que mejoren el ambiente universitario y la CVL ${ }^{(8)}$. Se pueden crear instancias de participación colectiva y diálogo ${ }^{(8)}$, y entre otras mejoras, crear espacios de convivencia para los profesores, disponer de un mobiliario ergonómico, comprar recursos materiales necesarios y mantener la infraestructura ${ }^{(26)}$.

El clima y las relaciones laborales también ha sido reconocido como un factor desgastante por parte de los enfermeros docentes, porque es mencionado como un factor primordial para promover las relaciones interpersonales e interprofesionales ${ }^{(26)}$. En este sentido, en el Departamento de Enfermería estudiado, la Comisión de Extensión tenía como función favorecer el buen clima laboral en la organización y por la cantidad de trabajo asumido, esta estrategia se adoptó en forma diferenciada por oficinas y subdirección. Así, las celebraciones de cumpleaños, navidad, fiestas patrias, nacimientos, funerales y conmemoraciones especiales se festejan dentro de la jornada laboral y son patrocinados por el Departamento. De la misma forma, se realiza el festejo del Día de la Enfermería y la finalización del ańo laboral, con la totalidad de los miembros de la unidad. 
Respecto a las estrategias personales, es saludable intervenir con estrategias de prevención y promoción de la salud, alentando la práctica de actividades físicas y de ocio ${ }^{(10)}$. Los trabajadores mencionan que cada persona tiene la responsabilidad en mejorar su CVL, cada trabajador tiene la necesidad de poner límites, manteniendo el equilibrio con el trabajo, de acuerdo con la realidad individual de cada uno, sin embargo, hay evidencias que los enfermeros desean tener una vida equilibrada, a pesar de que las cargas laborales asignadas sobrepasen las comprometidas $^{(9,14)}$. Existe la necesidad de mantener estrategias personales para evitar las amenazas del trabajo estresante como desconectarse del trabajo y no involucrarse ${ }^{(8)}$ más de lo necesario. La empresa o institución debe tener recursos laborales e individuales para favorecer la satisfacción laboral y con ello impactar en la CVL de los profesionales de enfermería ${ }^{(7)}$.

En este sentido, la Dirección actual del Departamento estudiado ha desarrollado un estudio de cargas de trabajo, de tal forma, ha contratado a los docentes necesarios para dar cumplimiento a la totalidad de las actividades, cubriendo el desarrollo de las actividades de Escuela y Departamento, referidas a la docencia, extensión e investigación. Aun así, el desarrollo de estas áreas en paralelo es visto como un desgaste por el académico, que repercute en la obligada dedicación a unas actividades más que a otras ${ }^{(32)}$, por lo que se considera fundamental, por ejemplo, se contraten personas que apoyen en el desarrollo de las actividades de docencia directa para liberar horas que necesariamente deben destinarse a investigación ${ }^{(15)}$.

Igualmente, de forma general, se verifica que las estrategias relatadas por los docentes son pertinentes $\mathrm{y}$ tienen el potencial de ser implementadas.

\section{REFERENCIAS}

1. Parra GD, Felli VEA. Work process of nursing professors. Rev Lat Am Enfermagem [Internet]. 2017 Dic [citado 16 ago 2020]; 25: e2946 Disponible en: 10.1590/1518-8345.1941.2946

2. Breilh J. Salud pública, universidad y poder. La universidad que queremos. Rev Fac Nac Salud Pública [Internet]. 2019 Ene [citado 16 ago 2020];
Finalmente, es necesario mencionar que la principal limitación de este estudio está en que solo se tiene la mirada de profesores de enfermería que se desempeñan en el ámbito docente público, siendo necesaria la perspectiva del área privada en el contexto del mercado educacional de la enfermería actual.

\section{CONCLUSIONES}

Los enfermeros y enfermeras que se desempeñan en docencia ven afectada su calidad de vida por factores laborales, de tal forma, la dialéctica en este trabajo precisa establecer estrategias de mejora desde la empresa y el individuo.

De esta manera, el desarrollo del presente estudio permitió captar los factores laborales que influyen en la calidad de vida: relaciones humanas favorables, ambiente favorable, bienestar-satisfacción y estrés. Así también, en relación a la construcción de estrategias para mejorarla, los trabajadores señalan, en primera instancia, que debe ser la institución quien debe establecer las condiciones para favorecer la CVL, a partir de las mejoras constantes derivadas de la política institucional, el sistema contractual y colectivo, la infraestructura, el clima y las relaciones laborales. En segunda instancia, es el trabajador quien debe implementar estrategias individuales.

Este estudio, que se centra en determinar una realidad determinada, a través de la mirada de la epidemiología crítica, entrega elementos para construir mejoras que contribuyan a la CVL de los enfermeros docentes, en un contexto de creciente número de escuelas y departamentos de enfermería que imparten la carrera en Chile y Latinoamérica.

1(37): 7-13. Disponible en: 10.17533/udea.rfnsp. v37n1aXX

3. Canales-Vergara M, Valenzuela-Suazo S, ParavicKlijn T. Condiciones de trabajo de los profesionales de enfermería en Chile. Enferm Univ [Internet]. 2016 Jul [citado 16 ago 2020]; 13(3): 178186. Disponible en: http://dx.doi.org/10.1016/j. reu.2016.05.004

4. De Oliveira ERA, Garcia ÁL, Gomes MJ, Bittar 
TO, Pereira AC. Gender and perceived quality of life: research with professors from the health area. Cienc Saude Coletiva [Internet]. 2012 Jun; 17(3): 741-747. Disponible en: http://www.scielo.br/pdf/ csc/v17n3/v17n3a21.pdf

5. Breilh J. El Debate Determinantes-Determinación: Aportes del Análisis Teórico, Epistemológico y Político. Rev Fac Nac Salud Pública [Internet]. 2013 Dic [citado 16 ago 2020]; 31(1): 13-27. Disponible en: http://www.scielo.org.co/pdf/rfnsp/ v31s1/v31s1a02.pdf

6. Hipólito MCV, Masson VA, Monteiro MI, Gutiérrez GL. Qualidade de vida no trabalho: avaliação de estudos de intervenção. Rev Bras Enferm [Internet]. 2017 Ene [citado 16 ago 2020]; 70(1): 189-197. Disponible en: http://dx.doi. org/10.1590/0034-7167-2015-0069

7. Orgambídez-Ramos A, Borrego-Alés Y. Apoyo social y engagement como antecedentes de la satisfacción laboral en personal de enfermería. Enferm Glob [Internet]. 2017 Oct [citado 16 ago 2020]; 16(48): 208-225. Disponible en: http:// dx.doi.org/10.6018/eglobal.16.4.260771

8. Silvério MR, Patrício ZM, Brodbeck IM, Grosseman S. O ensino na área da saúde e sua repercussáo na qualidade de vida docente. Rev Bras Educ Médica [Internet]. 2010 [citado 16 ago 2020]; 34(1): 65-73. Disponible en: http://www. scielo.br/pdf/rbem/v34n1/a08v34n1.pdf

9. Jamieson I, Kirk R, Andrew C. Work-life balance: What generation y nurses want. Nurse Lead. Nurse Leader [Internet], 2013 Jun [citado 16 ago 2020]; 11(3): 36-39. Disponible en: https://doi. org/10.1016/j.mnl.2013.01.010

10. De Oliveira JM, Santos PF, De Godoy FR, Assis MM, Cortez EA, Valente GSC. Riscos e doenças ocupacionais do docente universitário de enfermagem: implicaçóes na saúde do trabalhador. Rev Pesqui Cuid É Fundam Online [Internet]. 2013 Ene [citado 16 ago 2020]; 5(1): 3267-3275. Disponible en: 10.9789/2175-5361.2013v5n1p3267

11. Kuehn MB. Creating a healthy work environment for nursing faculty. Creat Nurs [Internet]. $2010 \mathrm{Nov}$ [citado 16 ago 2020]; 16(4): 193-197. Disponible en: http://www.ingentaconnect.com/content/springer/ crnu/2010/00000016/00000004/art00012

12. Dias MO, Souza NVDO, Penna LHG, Gallasch $\mathrm{CH}$. Percepção das lideranças de enfermagem sobre a luta contra a precarização das condiçóes de trabalho. Rev Esc Enferm USP [Internet]. 2019 Ago [citado 16 ago 2020]; 53: e03492 Disponible en: http://dx.doi.org/10.1590/s1980$220 \times 2018025503492$

13. Ortega MCB, Cecagno D, Llor AMS, De Siqueira $\mathrm{HCH}$, Montesinos MJL, Soler LM. Formación académica del profesional de enfermería y su adecuación al puesto de trabajo. Rev Lat Am Enfermagem [Internet]. 2015 May [citado 16 ago 2020]; 23(3): 404-410. Disponible en: 10.1590/0104-1169.0432.2569

14. Derby-Davis MJ. Predictors of nursing faculty's job satisfaction and intent to stay in academe. J Prof Nurs [Internet]. 2014 Ene [citado 16 ago 2020]; 30(1): 19-25. Disponible en: http://dx.doi. org/10.1016/j.profnurs.2013.04.001

15. Halcomb EJ, Andrew S, Peters K, Salamonson Y, Jackson D. Casualisation of the teaching workforce: implications for nursing education. Nurse Educ Today [Internet]. 2010 Ago [citado 16 ago 2020]; 30(6): 528-532. Disponible en: http://ro.uow.edu.au/cgi/ viewcontent.cgi?article $=1748 \&$ context $=$ smhpapers

16. Conceição MRD, Costa MS, De Almeida MI, Souza ÂMA, Cavalcante MBPT, Alves MDS. Quality of life for nurses when teaching professionaly: a study with the Whoqol-bref. Esc Anna Nery [Internet]. 2012 Jun [citado 16 ago 2020]; 16(2): 320-5. Disponible en: 10.1590/ S1414-81452012000200016

17. Luengo-Martínez CE, Sanhueza-Alvarado O. Formación del licenciado en Enfermería en América Latina. Aquichan [Internet]. 2016 Jun [citado 16 ago 2020]; 16(2): 240-255. Disponible en: http:// www.scielo.org.co/pdf/aqui/v16n2/v16n2a11.pdf

18. Luengo-Martínez C, Sanhueza O. Condiciones de trabajo y su relación con la calidad del cuidado y salud del profesional de enfermería. Med Segur Trab [Internet]. 2016 Oct [citado 16 ago 2020]; 62(245): 368-380. Disponible en: http://scielo. isciii.es/pdf/mesetra/v62n245/08_revision.pdf

19. Ramos CA. Los paradigmas de la investigación científica. Av En Psicol [Internet]. 2015 Ene [citado 16 ago 2020]; 23(1): 9-17. Disponible en: https:// doi.org/10.33539/avpsicol.2015.v23n1.167

20. Hernández LJ. Qué critica la epidemiología crítica: una aproximación a la mirada de Naomar Almeida Filho. Bol Obs en Salud [Internet]. 2009 Ago [citado 16 ago 2020]; 2(4): 18-28 Disponible en: http://www.revistas.unal.edu.co/index.php/bos/ article/download/10453/10943

21. Universidad de Chile. Departamento de Enfermería [Internet]. Facultad de Medicina: Santiago, Chile. 2010. Disponible en: http://www.medicina.uchile. $\mathrm{cl} /$ carreras/5010/enfermeria

22. Bardin L. Análise de Conteúdo. São Paulo: Ediçôes 70; 2011. 280 pp.

23. Guba E, Lincoln Y. Competencia de paradigmas en la investigación cualitativa. En: Dezin NK y Lincoln YS (Eds.). Handbook of Qualitative Research. California: Sage Publications. 1994; pp. 105-117.

24. Cruz AM, Almeida NG, Fialho AV, Rodrigues 
DP, Figueiredo JV, Oliveira AC. Percepção da enfermeira docente sobre sua qualidade de vida. Rev Rede Enferm Nordeste-Rev Rene [Internet]. 2015 [citado 16 ago 2020]; 16(3): 382-390. Disponible en: 10.15253/2175-6783.2015000300011

25. Sosa-Cerda OR, Cheverría-Rivera S, RodríguezPadilla ME. Calidad de vida profesional del personal de enfermería. Rev Enferm Inst Mex Seguro Soc [Internet]. 2010 Sep [citado 16 ago 2020]; 18(3): 153-58. Disponible en: http://www. medigraphic.com/pdfs/enfermeriaimss/eim-2010/ eim103f.pdf

26. Santos RS, Vaz DC, Alves DSB, Tocantins FR, Vianna MA, Silva TL. Diagnóstico de vulnerabilidades das docentes de uma escola de enfermagem: a influência do contexto. Rev Pesqui Cuid FundamOnline [Internet]. 2010 Abr [citado 16 ago 2020]; 2(2): 968-975. Disponible en: http://bases.bireme.br/cgi-bin/ wxislind.exe/iah/online/?IsisScript=iah/iah. $\mathrm{xis} \& \mathrm{src}=$ google $\&$ base $=\mathrm{BDENF} \&$ lang $=\mathrm{p} \&$ next Action=lnk\&exprSearch=22077\&indexSearch=ID

27. Bareño SJ, Berbesi FDY, Montoya ZCP. Risk factors associated with burnout among nursing staff Medellín-Colombia 2008. Investig Andina [Internet]. 2010 Sep [citado 16ago 2020]; 12(21):3648. Disponible en: http://www.scielo.org.co/scielo. php?pid=S0124-81462010000200004\&script $=$ sci_ arttext\&tlng=es

28. Suescún-Carrero S, Sarmiento G, Álvarez L, Lugo M. Calidad de vida laboral en trabajadores de una
Empresa Social del Estado de Tunja, Colombia. Rev Med Risaralda [Internet]. 2016 Ene [citado 16 ago 2020]; 22(1): 14-17. Disponible en: http:// www.scielo.org.co/pdf/rmri/v22n1/v22n1a03.pdf

29. Owens J. Life balance in nurse educators: A mixed-methods study. Nurs Educ Perspect [Internet]. 2017 [citado 16 ago 2020]; 38(4): 182188. Disponible en: https://journals.lww.com/ neponline/Abstract/2017/07000/Life_Balance_ in_Nurse_Educators_A_Mixed_Methods.5.aspx

30. Universidad de Chile. Plan de Desarrollo Institucional de la Universidad de Chile 2017 - 2026 [Internet]. Santiago, Facultad de Medicina, Universidad de Chile; 2016 [citado 16 ago 2020]. Disponible en: https://www.uchile.cl/portal/presentacion/senadouniversitario/documentos/30784/plan-de-desarrolloinstitucional-2017-2026

31. Gobierno de Chile. Ley 18.717, Artículo 4: Estatuto de salud, Asignaciones de colación y movilización [Internet]. 1998 Mar [citado 16 ago 2020]; pp. 1-10. Disponible en: file:///C:/Users/ denis/AppData/Local/Temp/LEY-18717_28MAY-1988.pdf

32. Leonello VM, De Campos OM. Educação superior em Enfermagem: o processo de trabalho docente em diferentes contextos institucionais. Rev Esc Enferm USP [Internet]. 2014 Dic [citado 16 ago 2020]; 48(6): 1093-1102. Disponible en: DOI: http://dx.doi.org/10.1590/S0080623420140000700018 